\title{
GAMMA RAYS FROM SOLAR FLARES
}

\author{
NATALIE MANDZHAVIDZE \\ Universities Space Research Association, \\ Lab. for High Energy Astrophysics, NASA/GSFC \\ AND \\ REUVEN RAMATY \\ Lab. for High Energy Astrophysics, NASA/GSFC \\ Greenbelt MD 20771, USA
}

\begin{abstract}
.
We review recent results obtained from the analysis of the solar flare gamma ray line emission: (a) The gamma ray derived ambient elemental abundances show that the First Ionization Potential (FIP) effect already sets in at relatively low altitudes in the solar atmosphere.

(b) The composition of the flare accelerated particles that produce the gamma rays exhibit heavy element and ${ }^{3} \mathrm{He}$ abundance enhancements that are typical for impulsive flares. Unlike the solar energetic particle (SEP) observations in interplanetary space, the gamma ray method allows us to trace the time development of these enhancements.

(c) Solar flare gamma ray spectroscopy provides the most direct measure of the abundances of the two very high FIP elements, $\mathrm{He}$ and $\mathrm{Ne}$, in subcoronal regions leading to somewhat higher abundances than the generally accepted values.

(d) The high intensities of the $\alpha \alpha$ lines observed from a number of flares imply a high $(\gtrsim 0.1)$ ambient $\mathrm{He} / \mathrm{H}$ and/or accelerated $\alpha / \mathrm{p}$.

(e) There are indications for the isotopic fractionation of He from the photosphere to corona that has important implications on the mechanism of solar wind acceleration, the protosolar deuterium abundance and Galactic chemical evolution.
\end{abstract}

\section{Introduction}

Solar flare gamma ray emission is produced by accelerated electrons and ions interacting with the ambient solar atmosphere. The main components of this emission are: electron bremsstrahlung, which dominates at energies $\lesssim 1 \mathrm{MeV}$ and $\sim 10-50 \mathrm{MeV}$; nuclear gamma ray line emission $(\sim 1-10$ $\mathrm{MeV}$ ) and pion decay emission ( $\gtrsim 50 \mathrm{MeV}$ ). Gamma rays were observed from many flares with detectors on SMM, CGRO, GAMMA-1, GRANAT and Yohkoh (see [1]) and they provide important information on many astrophysical problems, including solar atmospheric dynamics, solar flare and solar wind particle acceleration and even the chemical evolution of the Galaxy.

\section{Solar Atmosphere: First Ionization Potential (FIP) Effect}

The fluences of narrow gamma ray lines observed from 19 flares with SMM/GRS were presented recently [2]. These narrow lines result from nuclear interactions of flare accelerated protons and $\alpha$-particles with heavier nuclei in the solar atmosphere and can be used to determine ambient elemental abundances [3]. In Fig. 1 we show the gamma ray derived low FIP $(<10 \mathrm{eV})$ to high FIP $(>10 \mathrm{eV})$ element abundance ratios for $19 \mathrm{SMM}$ flares together with the average photospheric and coronal abundance ratios. It is clear that the FIP bias, that is manifested by $\sim 4-5$ times higher low FIP/high FIP element abundance ratios in the corona relative to the photosphere [5], already sets in at relatively low altitudes in the solar atmosphere (low chromosphere) where the nuclear 

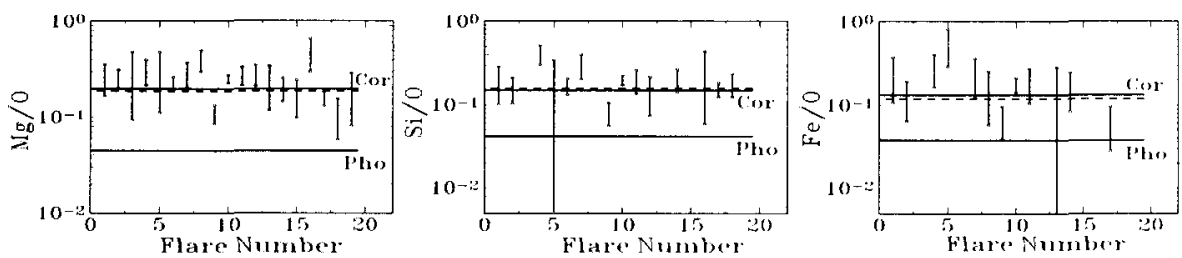

Figure 1. Vertical bars: Low FIP to high FIP element abundance ratios determined [3] for 19 flares. Horizontal lines: solid - coronal and photospheric abundance ratios [6]; dashed lines - best fitting values to the gamma ray derived abundance ratios.

interactions and the gamma ray production takes place. This provides important new information on solar atmospheric dynamics and should help to better understand the origin of the FIP effect.

\section{Solar Flare Particle Acceleration}

The two classes of solar flares (impulsive and gradual) are formally distinguished according to the duration of the accompanying soft X-ray emission, but the most significant differences are seen in the composition of the accelerated particles [6]. In gradual flares (GF) the accelerated particle composition is identical to that of the ambient corona, while impulsive flares (IF) are characterized by very specific abundance anomalies. The most dramatic signature of IFs is the ${ }^{3} \mathrm{He}$ abundance enhancement, ${ }^{3} \mathrm{He} /{ }^{4} \mathrm{He}$ can reach values that are $10^{4}$ times higher than the ambient ${ }^{3} \mathrm{He} /{ }^{4} \mathrm{He}$. Also enhanced are heavy elements, Fe by about factor of $10, \mathrm{Ne}, \mathrm{Mg}, \mathrm{Si}$ and $\mathrm{S}$ by about factor of 3 relative to $\mathrm{C}, \mathrm{N}, \mathrm{O}$. These abundance anomalies imply that in impulsive flares particles are accelerated due to gyroresonant interactions with various modes of plasma waves, while particles from gradual flares are accelerated directly from the ambient coronal material by CME driven shocks (e.g. [7]).

Independent information on the composition of flare accelerated particles can be obtained from the broad gamma ray lines that result from the nuclear interactions of accelerated heavy ions with ambient $\mathrm{H}$ and $\mathrm{He}$. Unlike narrow lines, the broad lines cannot be resolved individually. However, the fact that the gamma ray lines from heavy elements $(\mathrm{Ne}, \mathrm{Mg}, \mathrm{Si}, \mathrm{S}, \mathrm{Fe}$ ) are mostly concentrated in the $\sim 0.8-2 \mathrm{MeV}$ range, while the $\sim 4-7 \mathrm{MeV}$ range is dominated by nuclear lines from $\mathrm{C}, \mathrm{N}, \mathrm{O}$, allows the determination of the relative abundances of elements in these two groups ( $\mathrm{Ne}-\mathrm{Fe} / \mathrm{C}-\mathrm{O})$. Using the ratio of gamma ray emissions in these two energy bands observed with PHEBUS/GRANAT from the behind the limb flare on 1991 June 1, we found very significant heavy element enhancements for the interacting accelerated particles [8]. Furthermore, these enhancements kept increasing with time and towards the end of the flare they reached the highest enhancements observed in space during impulsive flares. Interestingly, this increase takes place at the time when the acceleration efficiency itself starts to decrease as is evident from the gradual decrease of the gamma ray emission and (possibly) the steepening of the accelerated particle spectrum.

We also found that, similar to the particles from IF, the particles that produce gamma rays have high ${ }^{3} \mathrm{He} /{ }^{4} \mathrm{He}[4]$ and high electron to proton ratios [9]. These properties of the interacting particles seem to be independent of the flare type, they appear in both the IF and GF that we studied. Recently we suggested [10] a method of determining the accelerated ${ }^{3} \mathrm{He} /{ }^{4} \mathrm{He}$ by spectrally resolving the gamma ray line complex around $\sim 1 \mathrm{MeV}$ which contains lines produced by accelerated ${ }^{3} \mathrm{He}$ at $0.937,1.04$ and $1.08 \mathrm{MeV}$. An important advantage of using the gamma ray method for determining the accelerated heavy element and ${ }^{3} \mathrm{He}$ abundances is its ability to provide time resolution, i.e. to follow the element abundances and their enhancements with time. This is basically equivalent to tracing the development of particle acceleration on a real time scale. In contrast, direct SEP observations in space can only provide information on the element abundances that are integrated over an entire particle event, typically lasting for many hours. In addition various propagation effects may mask the properties of the flare accelerated particles observed near the earth.

Another important result of our studies [3] is the finding that there is approximate equipartition between the energies imparted into accelerated electrons and ions, contrary to the previous paradigm that the bulk of the flare energy resides in the nonthermal electrons. 

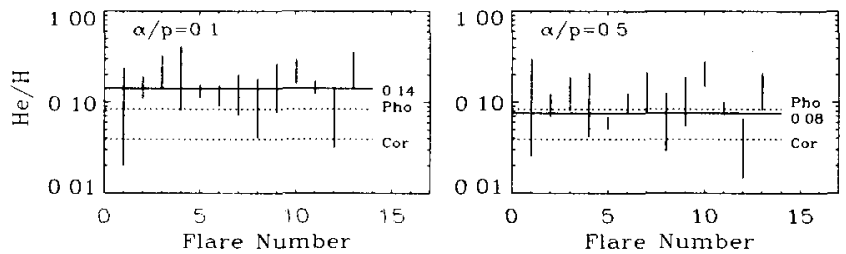

Figure 2. Vertical bars: $\mathrm{He} / \mathrm{H}$ derived for 13 flares using coronal $\mathrm{O} / \mathrm{H}=5.26 \times 10^{-4}$. Horizontal lines: dotted - corona (0.04) and photosphere $(0.084)$; solid - best fitting values to the gamma ray derived $\mathrm{He} / \mathrm{H}$.

\section{Helium}

Nuclear interactions of flare accelerated $\alpha$-particles with ambient $\mathrm{He},{ }^{4} \mathrm{He}(\alpha, \mathrm{p})^{7} \mathrm{Li}^{\star 0.478}$ and ${ }^{4} \mathrm{He}(\alpha, \mathrm{n}){ }^{7} \mathrm{Be}^{\star 0.429}$, result in two gamma ray lines which due to Doppler broadening blend into a broad feature from $\sim 0.35$ to $\sim 0.55 \mathrm{MeV}$, usually referred to as the $\alpha \alpha$ line [11]. Using the intensities of this line together with the $6.13 \mathrm{MeV}{ }^{16} \mathrm{O}$ deexcitation line observed with SMM/GRS [12] we determined [10] the ambient $\mathrm{He} / \mathrm{O}$ abundance ratio for 13 flares. By combining the gamma ray derived values of $\mathrm{He} / \mathrm{O}$ with the ambient $\mathrm{O} / \mathrm{H}$ we obtained $\mathrm{He} / \mathrm{H}$ in the gamma ray production region. We used the solar wind derived coronal $\mathrm{O} / \mathrm{H}=5.26 \times 10^{-4}[15]$, which is lower than the photospheric value of $7.41 \times 10^{-4}$ [16]. The results are shown in Fig. 2 together with the solar wind [15] and SEP [6] derived coronal values of $\sim 4 \%$ and the photospheric value of $\sim 8.4 \%$ determined from helioseismology [13]. The later agrees with the values predicted by the standard solar model when heavy element diffusion is taken into account [14]. Together with the ambient $\mathrm{He}$ abundance, the $\alpha \alpha$ line intensity also depends on the accelerated $\alpha$-particle to proton ratio $(\alpha / \mathrm{p})$, which we use as a parameter. In Fig. 2 we show results for two values of $\alpha / \mathrm{p}$ : the standard value of 0.1 and a higher value, $\alpha / \mathrm{p}=0.5$. We see that for both values of $\alpha / \mathrm{p}$ the gamma ray derived $\mathrm{He} / \mathrm{H}$ is significantly higher than the coronal value. For $\alpha / \mathrm{p}=0.1$ it is also higher than $\mathrm{He} / \mathrm{H}$ in the photosphere, while for $\alpha / \mathrm{p}=0.5$ it is consistent with the photospheric He/H. Thus, in order to account for the observed $\alpha \alpha$ line fluences, either the accelerated $\alpha / \mathrm{p}$ or the ambient $\mathrm{He} / \mathrm{H}$ has to be high. Note, that if we had used the photospheric $\mathrm{O} / \mathrm{H}$, even higher values of $\mathrm{He} / \mathrm{H}$ or $\alpha / \mathrm{p}$ would be needed in order to account for the data. Both options (high $\mathrm{He} / \mathrm{H}$ or high $\alpha / \mathrm{p}$ ) are acceptable and they both have serious implications. The role of high subcoronal helium abundance in stabilizing the solar wind flux, as well as dynamic models of the outer solar atmosphere in which the effects of diffusion and gravitational settling can lead to He abundance enhancements, have been considered in a number of theoretical papers (e.g. [17]). These effects could account for the episodes of high $\mathrm{He} / \mathrm{H}$ that are sometimes seen in the highly variable equatorial solar wind. On the other hand, high values of $\alpha / \mathrm{p}$ have important implications on the theory of the solar flare particle acceleration [7]. Although the current gamma ray data do not allow us to distinguish between the two possibilities, future observations with high resolution germanium detectors will allow us to resolve this issue by measuring narrow gamma ray lines at $0.339,1.00,1.05$ and $1.19 \mathrm{MeV}$ that are produced exclusively by accelerated $\alpha$-particles (no contribution from protons) [10]. This will allow us to unambiguously determine the accelerated $\alpha / \mathrm{p}$ and ultimately the ambient $\mathrm{He} / \mathrm{H}$.

Earlier we found that in the gamma ray production region $\mathrm{Ne} / \mathrm{O}$ is higher than in the corona [3]. Similar to $\mathrm{He}$, in the photosphere $\mathrm{Ne}$ is not measured spectroscopically, its abundance is inferred from HII regions and hot stars [5]. We believe that solar flare gamma ray spectroscopy provides the most direct measure of the abundances of the two very high FIP elements $\left(\mathrm{FIP}_{\mathrm{He}}=\mathbf{2 4 . 4 6}\right.$; $\mathrm{FIP}_{N e}=21.47$ ) in subcoronal regions, suggesting the $\mathrm{Ne} / \mathrm{O}$ and $\mathrm{He} / \mathrm{O}$ that are higher than both their coronal and the generally accepted photospheric values.

\section{He Isotopic Ratio}

The consistent ${ }^{3} \mathrm{He} /{ }^{4} \mathrm{He}$ values of $(4.25 \pm 0.21) \times 10^{-4}[18],(4.88 \pm 0.48) \times 10^{-4}[15]$ and $(4.4 \pm$ $0.4) \times 10^{-4}[19]$ were obtained from solar wind observations with Apollo, ISEE-3 and Ulysses respectively. These can be compared with the photospheric ${ }^{3} \mathrm{He} /{ }^{4} \mathrm{He}$ determined from solar flare gamma ray line data. The time delays of the $2.223 \mathrm{MeV}$ line, that results from the neutron capture 
on photospheric $\mathrm{H}$, relative to prompt deexcitation gamma ray lines allow the determination of the photospheric ${ }^{3} \mathrm{He} / \mathrm{H}$. Applying this method to the gamma ray data from one flare, it was found that ${ }^{3} \mathrm{He} / \mathrm{H}=(2.3 \pm 0.8) \times 10^{-5}$ and ${ }^{3} \mathrm{He} / \mathrm{H}=(1.8 \pm 0.8) \times 10^{-5}$ for the two different assumed angular distributions of the accelerated particles [20]. Combining these values with the photospheric ${ }^{4} \mathrm{He} / \mathrm{H}=0.084$ we obtain ${ }^{3} \mathrm{He} /{ }^{4} \mathrm{He}=(2.7 \pm 0.9) \times 10^{-4}$ and ${ }^{3} \mathrm{He} /{ }^{4} \mathrm{He}=(2.1 \pm 0.9) \times 10^{-4}$ which are lower than the solar wind derived coronal values. This suggests the isotopic fractionation of helium. Since the FIPs of ${ }^{3} \mathrm{He}$ and ${ }^{4} \mathrm{He}$ are very close, this fractionation must be caused by the acceleration of the solar wind, not material transport from the photosphere to corona. Obviously, this is important new information for the understanding of the mechanism of solar wind acceleration. However, the problem of the photospheric ${ }^{3} \mathrm{He} /{ }^{4} \mathrm{He}$ requires more work, namely we need to study the gamma ray data from more flares and to assess the uncertainties related to the accelerated particle composition and the solar atmospheric model.

\section{Protosolar Deuterium}

The protosolar abundance of deuterium can be related [21] to the present day solar helium isotopic ratio ${ }^{3} \mathrm{He} /{ }^{4} \mathrm{He}$ by the expression $\left({ }^{2} \mathrm{H} / \mathrm{H}\right)_{p}=2.675 \times 10^{-5}+\left(9.364 \times 10^{-2}-2.452 x\right) x$, where $x={ }^{3} \mathrm{He} /{ }^{4} \mathrm{He}-4.0 \times 10^{-4}$. Taking ${ }^{3} \mathrm{He} /{ }^{4} \mathrm{He}=(2.7 \pm 0.9) \times 10^{-4}$, which is the highest among the two values obtained in the previous section, we find $\left({ }^{2} \mathrm{H} / \mathrm{H}\right)_{p}=(1.45 \pm 0.48) \times 10^{-5}$. This is significantly lower than Jovian value of $(5 \pm 2) \times 10^{-5}$ measured with the Galileo atmospheric space probe [22]. In [21] the authors used the solar wind value of ${ }^{3} \mathrm{He} /{ }^{4} \mathrm{He}$ and also found lower than Jovian value of $\left({ }^{2} \mathrm{H} / \mathrm{H}\right)_{p}=(3.01 \pm 0.17) \times 10^{-5}$, which they attributed to the incorporation into Jupiter of ${ }^{2} \mathrm{H}$ enriched ices from the protosolar cloud. Since the gamma ray data suggest that the ${ }^{3} \mathrm{He}$ abundance in the photosphere is lower than in the corona, the discrepancy between the protosolar and the Jovian ${ }^{2} \mathrm{H} / \mathrm{H}$ becomes even greater, thereby strengthening the need for such ${ }^{2} \mathrm{H}$ enriched ices. The gamma ray derived $\left({ }^{2} \mathrm{H} / \mathrm{H}\right)_{p}$ can also be compared with the present day deuterium abundance in the interstellar medium $\left({ }^{2} \mathrm{H} / \mathrm{H}\right)_{I S M}=(1.47-1.72) \times 10^{-5}[23]$, thereby providing one more important milestone in the understanding of Galactic chemical evolution.

\section{References}

1. Ramaty, R. \& Mandzhavidze N. (1996) High Energy Solar Physics, eds. R. Ramaty, N. Mandzhavidze \& X.-M. Hua, AIP Conf. Proc. 374, (AIP, New York), 533

2. Share, G.H. \& Murphy, R.J. (1995) $A p J, 452,933$

3. Ramaty, R., Mandzhavidze N., Kozlovsky, B. \& Murphy, R.J. (1995) ApJ, 445, L193

4. Ramaty, R., Mandzhavidze N. \& Kozlovsky, B. (1996) High Energy Solar Physics, eds. R. Ramaty, N Mandzhavidze \& X.-M. Hua, AIP Conf. Proc. 374, (AIP, New York), 172

5. Meyer, J.-P. (1996) Cosmic Abundances, eds. S.S. Holt \& G. Sonneborn, ASP Conf. Series 99, (ASP, San Francisco), 127

6. Reames, D.V. (1995) Adv. Space Res., 15, (7) 41

7. Miller, J.A., et al. (1997) JGR, 102, A7, 14631

8. Ramaty, R., Mandzhavidze, N., Barat, C. \& Trottet, G. (1997) ApJ, 479, 458

9. Ramaty, R., Mandzhavidze, N., Kozlovsky, B. \& Skibo, J.G. (1993) Adv. Space Res. 13, (9) 275

10. Mandzhavidze, N., Ramaty, R. \& Kozlovsky, B. (1997) ApJ Letters, in press (November 1 issue)

11. Kozlovsky, B. \& Ramaty, R. (1974) ApJ, 191, L43

12. Share, G.H. \& Murphy, R.J. (1997) $A p J$, 485, 409

13. Hernandez, F.P. \& Christensen-Dalsgaard, J. (1994) Mon. Not. R. Astron. Soc., 169, 475

14. Bahcall, J.N. \& Pinsonneault, M.H. (1995) Rev. Mod. Phys., 67, 781

15. Coplan, M.A., Ogilvie, K.W., Bochsler, P. \& Geiss, J. (1990) Solar Phys., 128, 195

16. Grevesse, N., Noels, A. \& Sauval, A.J. (1996) Cosmic Abundances, eds. S.S. Holt, \& G. Sonneborn, ASP Conf. Series, 99, (ASP, San Francisco), 117

17. Hansteen, V.H., Leer, E. \& Holzer, T.E. (1997) ApJ, 482, 498

18. Geiss, J., et al. (1972) Appolo Preliminary Science Report, NASA SP-315, section 14

19. Bodmer, R., Bochsler, P., Geiss, J., Von Steiger, G. \& Gloeckler, G. (1994) Space Sci. Rev., 72, 61

20. Hua, X-M. \& Lingenfelter, R.E. (1987) Solar Phys. 107, 351

21. Gautier, D. \& Morel, P. (1997) $A B A, \mathbf{3 2 3}$, L9

22. Nieman, H.B., et al. (1996) Science, 272, 846

23. Linsky, J.L., et al. (1993) $A p J, 402,694$ 